\title{
Covid-19, desinformação e Facebook: circulação de URLs sobre a hidroxicloroquina em páginas e grupos públicos ${ }^{1}$
}

\section{Felipe Bonow Soares'}

https://orcid.org/0000-0003-4850-9255

\section{Paula Viegas"}

https://orcid.org/0000-0002-6038-6502

\section{Carolina Bonoto"}

https://orcid.org/0000-0003-2184-7625

\section{Raquel Recuero"II}

https://orcid.org/0000-0002-7417-9782

I - MIDIARS.

Pelotas (RS). Brasil.

II - Universidade Federal do Rio Grande do Sul. Porto Alegre (RS). Brasil.

III - Universidade Federal de Pelotas.

Pelotas (RS). Brasil.

Universidade Federal do Rio Grande do Sul.

Porto Alegre (RS). Brasil. 
Resumo: Neste artigo, analisamos a circulação de URLs desinformativas no Facebook. Nosso estudo de caso é o uso da hidroxicloroquina como cura ou profilaxia para o Covid-19. Analisamos a presença de URLs em mais de 70 mil publicações de páginas e grupos públicos no Facebook. Utilizamos a Análise de Redes Sociais para mapear a circulação das URLs em redes de páginas e grupos e a Análise de Conteúdo para identificar as fontes das informações e a presença de desinformação. Nossos resultados mostram que (1) a circulação de URLs foi polarizada, tanto no contexto de páginas, quanto grupos; (2) o conteúdo desinformativo foi utilizado para reforçar uma narrativa pró-hidroxicloroquina; e (3) os grupos deram maior visibilidade a mídias hiperpartidárias e desinformação em comparação com as páginas, e os grupos também registraram maior frequência no compartilhamento de URLS.

Palavras-chave: circulação de informações; Covid-19; desinformação; Facebook; polarização.

\begin{abstract}
Covid-19, disinformation and Facebook: circulation of URLs about hydroxychloroquine in public pages and groups - In this paper, we analyze the circulation of disinformation URLs on Facebook. Our case study is the use of hydroxychloroquine as a cure or prophylaxis for Covid-19. We analyze the presence of URLs in over 70 thousand posts in public pages and groups on Facebook. We use Social Network Analysis to map URLs circulation in pages and group networks. We use Content Analysis to identify the sources of information and the presence of disinformation. Our results are: (1) URLS circulation were polarized, both in pages and group networks; (2) disinformation was used to reinforce a pro-hydroxychloroquine narrative; and (3) groups gave more visibility to hyperpartisan media and disinformation, compared to the pages; groups also more often shared URLs.
\end{abstract}

Keywords: information circulation; Covid-19; disinformation; Facebook; polarization.

\title{
Introdução
}

Em dezembro de 2019 surgiram os primeiros casos de Covid-19 em humanos. Em pouco tempo, a doença se espalhou pelo mundo, com o primeiro caso confirmado no Brasil em fevereiro de 2020. Em função do impacto da pandemia no mundo, diversos testes foram realizados em busca de tratamentos mais efetivos. Neste contexto, a hidroxicloroquina apresentou resultados promissores em pequenos estudos iniciais, porém, mostrou-se ineficaz em estudos posteriores ${ }^{2}$. Ainda assim, em março de 2020, Donald Trump, presidente dos Estados Unidos, apontou o medicamento como 
a esperança no tratamento do Covid-19². Em seguida, Jair Bolsonaro (sem partido), presidente do Brasil, ecoou a declaração e passou a tratar a hidroxicloroquina como "cura" para a doença, inclusive aumentando a produção da droga no Brasil".

Estudos científicos que analisaram o potencial de uso da hidroxicloroquina no tratamento de Covid-19 não encontraram evidências de eficácia no uso do medicamento, ainda assim, o medicamento foi apontado como suposta cura ou método profilático no tratamento da doença, com base em informações falsas publicadas nas mídias sociais (ARAÚJO; OLIVEIRA, 2020). Esse tipo de conteúdo, distorcido, manipulado ou fabricado, que tem a função de enganar, é o que chamamos desinformação (FALLIS, 2015). A discussão sobre a droga foi mobilizada por meio do discurso político, já que o político Jair Bolsonaro teve papel central na circulação de desinformação sobre o tema quando se manifestou a favor do medicamento (RECUERO; SOARES, 2020). Com isso, campanhas desinformativas com o objetivo apoiar o presidente passaram a compartilhar conteúdo que apoiava a utilização (RECUERO, SOARES; ZAGO, 2020).

O caso da desinformação sobre a hidroxicloroquina se enquadra no que a Organização Mundial da Saúde (OMS) chamou de "infodemia", causada pelo excesso de informações sobre o tema, as disputas de sentido e a circulação de desinformação ${ }^{5}$. Como destacado pela OMS, a infodemia é um problema no combate ao Covid-19 porque pode ampliar o número de casos, já que atrapalha a ação coordenada dos indivíduos no combate ao vírus. A infodemia é fortalecida pela polarização política, já que os sentimentos relacionados ao Covid-19 espelham essa polarização e seguem alinhamentos políticos (ALLCOTT et al., 2020; GRUZD; MAI, 2020; RECUERO; SOARES, 2020; RECUERO, SOARES; ZAGO, 2020).

Neste contexto, focamos na circulação de desinformação sobre a hidroxicloroquina no Facebook. Nossa pergunta de pesquisa é: como circulou o conteúdo a respeito do medicamento nos grupos e páginas do Facebook? Escolhemos o Facebook porque a plataforma é utilizada por mais de três

3 Disponível em: <https://abcnews.go.com/Health/timeline-tracking-trump-alongside-scientific-developments-hydroxychloroquine/story?id=72170553>. Acessado em 15 nov.2020.

4 Disponível em: <https://www1.folha.uol.com.br/equilibrioesaude/2020/03/bolsonaro-manda-exercito-produzir-mais-cloroquina-mesmo-sem-acao-comprovada-contra-covid-19.shtml>. Acessado em 15 nov.2020.

5 Disponível em: <https://www.who.int/teams/risk-communication/infodemic-management/>. Acessado em 15 nov.2020. 
quartos dos brasileiros ${ }^{6}$ que participaram do relatório de notícias digitais do Reuters Institute, que também apontaram o Facebook como a principal mídia social na busca por notícias (NEWMAN et al., 2020). Para a análise, coletamos mais de 70 mil publicações em grupos e páginas públicas do Facebook por meio do CrowdTangle (2020). Como critério para essa coleta, selecionamos apenas publicações com URLs e que continham os termos "hidroxicloroquina" ou "cloroquina" + "covid" ou "coronavírus". Para a análise destes dados, utilizamos a Análise de Redes Sociais para mapear a circulação das URLs e Análise de Conteúdo para identificar as fontes de informação dos links mais compartilhados e a presença de desinformação, Dentro dessa proposta, os nossos objetivos foram: (1) mapear a circulação de conteúdo relacionado a hidroxicloroquina no Facebook, (2) identificar e caracterizar o conteúdo desinformativo sobre o tema, (3) analisar as diferenças na circulação de conteúdo nas páginas e grupos públicos.

\section{Desinformação, polarização e Covid-19}

Chamamos de desinformação o conteúdo que é distorcido, manipulado ou inteiramente falso e tem a função de enganar (FALLIS, 2015). A desinformação pode ser uma informação completamente fabricada, por exemplo, mas é geralmente produzida a partir de distorções, em que contextos falsos são associados a informações verdadeiras, que chamamos de conteúdo enganoso, no qual é criado um contexto falso para um conteúdo verdadeiro, uma associação falsa entre dois conteúdos verdadeiros, ou mesmo uma associação entre um conteúdo verdadeiro e um falso (WARDLE; DERAKHSHAN, 2017). A desinformação enganosa é mais dificilmente desmentida, pois frequentemente é explicitada como uma "história alternativa" ou uma "outra visão" dos fatos.

A produção de desinformação é também intencional, produzida para enganar. Não é um "erro honesto", mas um conteúdo criado intencionalmente, geralmente visando algum ganho para alguém, muitas vezes, político (BENKLER, FARIS; ROBERTS, 2018). No caso da desinformação sobre o Covid-19, também há frequentemente um enquadramento político para o conteúdo, que é muitas vezes impulsionado por redes políticas (RECUERO; SOARES, 2020).

A desinformação sobre o Covid-19 tem sido utilizada, por exemplo, para criticar autoridades públicas e culpar atores políticos pelo espalhamento

6 O Brasil tem aproximadamente 120 milhões de usuários ativos na plataforma. Disponível em: <https://www.tecmundo.com.br/redes-sociais/153570-brasil-4-pais-usuarios-facebook-quarentena.html>. Acessado em 15 de novembro de 2020. 
do vírus (BRENNEN, SIMON; NIELSEN, 2020). Pesquisas também têm mostrado que atores políticos frequentemente estão relacionados à disseminação de conteúdos falsos, como, por exemplo, no caso da hashtag \#FilmYourHospital7, utilizada no contexto dos Estados Unidos e posteriormente adotada no Brasil para afirmar que a pandemia de Covid-19 não passava de uma mentira (GRUZD; MAl, 2020). Outro exemplo foi o documentário "Plandemic"8, espaIhado rapidamente na rede, que afirmava que a pandemia seria uma farsa ou uma ação planejada, em que o vírus teria sido criado por humanos (KEARNEY, CHIANG; MASSEY, 2020). Na Europa, figuras políticas auxiliaram na circulação de informações distorcidas relacionadas com o uso de Ibuprofeno (XAUDIERA; CARDENAL, 2020).

No contexto brasileiro, a campanha pelo uso da hidroxicloroquina foi um dos principais conteúdos desinformativos que circularam na mídia social (ARAÚJO; OLIVEIRA, 2020). Ali, esse tipo de desinformação foi associado ao discurso político (RECUERO; SOARES, 2020) e à polarização (RECUERO, SOARES; ZAGO, 2020). Assim, a polarização política também tem efeitos no espalhamento de conteúdo sobre o Covid-19 e na forma como as pessoas reagem à pandemia (ALLCOTT et al., 2020).

A polarização ocorre, basicamente, quando dois grupos possuem visões opostas sobre uma temática (BARBERÁ, 2020). No contexto das mídias sociais, a polarização pode favorecer "dietas midiáticas" distintas, ou seja, que os grupos se informem a partir de fontes diferentes conforme seus posicionamentos nas discussões (BENKLER, FARIS; ROBERTS, 2018; RECUERO, SOARES; ZAGO, 2020). Assim, a polarização pode favorecer a centralidade de mídias hiperpartidárias e o espalhamento da desinformação nas mídias sociais (RECUERO, SOARES; GRUZD, 2020).

Mídias hiperpartidárias são veículos, frequentemente nativos de plataformas digitais, que produzem conteúdo que favorece uma perspectiva ideológica em particular, geralmente dando um enquadramento político no conteúdo que produzem (BENKLER, FARIS; ROBERTS, 2018). Assim, os veículos hiperpartidários produzem versões "alternativas" dos fatos e se apresentam como contraponto do jornalismo tradicional (LARSSON, 2019). Em função de tais características, as mídias hiperpartidárias estão frequentemente associadas

7 Disponível em: <https://socialmedialab.ca/2020/04/24/hospitals-around-the-world-are-being-targeted-by-conspiracy-theorists/>. Acessado em 15 de novembro de 2020.

8 Disponível em: <https://projetocomprova.com.br/publica\%C3\%A7\%C3\%B5es/video-plandemic-faz-afirmacoes-falsas-sobre-a-covid-19/>. Acessado em 15 de novembro de 2020. 
ao espalhamento de desinformação (RECUERO, SOARES; GRUZD, 2020) ${ }^{9}$. Além disso, o contexto polarizado das conversações pode contribuir para a emergência de grupos com "dietas midiáticas" distintas e também para a polarização assimétrica, que ocorre quando um grupo político tem acesso a informações variadas e o outro compartilha desinformação em maior frequência (BENKLER, FARIS; ROBERTS, 2018). Portanto, o contexto polarizado tem impacto na forma de propagação de informações nas mídias sociais

\section{Circulação de informações nas mídias sociais}

As mídias sociais são espaços que permitem fluxos comunicacionais mais horizontalizados em função de suas dinâmicas estruturais e das características de seus públicos (BOYD, 2011; RECUERO, BASTOS; ZAGO, 2015). Deste modo, a circulação de informações nesses espaços é dependente da ação de usuários, ou seja, para que o conteúdo circule nessas plataformas, que os atores interajam, curtam, comentem ou repliquem o mesmo (ZAGO; BASTOS, 2013). O processo de circulação de informações também é influenciado pelos algoritmos das mídias sociais, que mobilizam um processo de filtragem social que podem dar preferência a um conteúdo ao invés de outro a partir de lógicas de datificação das plataformas (GILLESPIE, 2018). Além disso, as próprias ferramentas utilizadas pelos usuários no compartilhamento de conteúdo (por exemplo, compartilhar no Facebook e retweet no Twitter) são criadas pelas plataformas com base nos seus modelos de negócio (D'ANDRÉA, 2020). Assim, o processo de circulação de informações nas plataformas de mídias sociais é dependente da ação dos usuários, mas também influenciado pelas lógicas das plataformas e seus modelos de negócio.

As dinâmicas na circulação de informações influenciam a forma como ocorrem as discussões políticas nas mídias sociais, que tendem a assumir estruturas divididas, nas quais existem dois grupos com posições antagônicas e com poucas conexões entre si (HIMELBOIM et al., 2017), em uma estrutura polarizada, pois os grupos tendem a circular apenas aquele conteúdo com o qual concordam (BENKLER, FARIS; ROBERTS, 2018; RECUERO, SOARES; ZAGO, 2020). Assim, o que circula em um grupo não circula no outro. Esse processo pode, por exemplo, favorecer a exposição seletiva a conteúdo político, quando usuários consomem preferencialmente conteúdo que favorece sua visão política (STROUD, 2010). Essa exposição seletiva é motivada pelas escolhas

9 Discussão semelhante foi realizada no artigo de Soares et al. (2020), publicado em: <http://www. intercom.org.br/sis/eventos/2020/resumos/R15-0550-1.pdf>. 
dos próprios usuários no processo de seleção de informações nas mídias sociais (BAKSHY, MESSING; ADAMIC, 2015) e também pelas dinâmicas das plataformas (GILLESPIE, 2018; D'ANDRÉA, 2020).

A circulação de informações e a participação dos usuários neste processo também depende das estruturas das mídias sociais e das dinâmicas de seus públicos (BOYD, 2011). Assim, duas questões são importantes para tal processo: as affordances das plataformas e a maneira como os usuários percebem os espaços de interação. De forma geral, o conceito de affordances se refere ao processo dialógico entre as funcionalidades técnicas das plataformas (suas ferramentas, o que permitem ou não fazer etc.) e as apropriações dos usuários (as formas como utilizam as funcionalidades técnicas das plataformas) (EVANS et al., 2017). Isto é, a noção de affordances se refere às materialidades das plataformas, suas ferramentas e funcionalidades, e o seu efeito nas práticas dos usuários, que acabam moldadas e padronizadas por essa materialidade (D'ANDRÉA, 2020). Assim, as affordances estão relacionadas com os artefatos tecnológicos, a maneira como informam como devem ser utilizados e seus possíveis usos (BUCHER; HELMOND, 2018). Essas ferramentas possuem formas limitadas de apropriação, mas também podem ser utilizadas pelos usuários de maneiras não planejadas pelas mesmas.

Além disso, a maneira como os usuários percebem os espaços de interações afeta a forma como compartilham conteúdo. Valeriani e Vaccari (2018) identificaram que usuários modificam seus comportamentos conforme a plataforma utilizada na interação. Isso porque muitas vezes compartilham certos conteúdos em espaços mais privados (como aplicativos de mensagens) que não compartilhariam em outras plataformas mais públicas. Nesse sentido, interessa-nos observar como ocorreram os compartilhamentos de URLS em páginas públicas e grupos públicos no Facebook, já que são contextos que mobilizam diferentes affordances da plataforma e podem ser afetados também pela forma como os usuários percebem cada espaço.

\section{Método}

Como dito, nosso objetivo é analisar a circulação de desinformação sobre a hidroxicloroquina no Facebook. Tal objetivo central desdobra-se em três específicos: (1) mapear a circulação de conteúdo relacionado a hidroxicloroquina no Facebook; (2) identificar e caracterizar o conteúdo desinformativo sobre o tema; (3) analisar as diferenças na circulação de conteúdo nas páginas e grupos públicos. 
Utilizando o CrowdTangle (2020) ${ }^{10}$, coletamos publicações de páginas e grupos públicos do Facebook entre $1^{\circ}$ de março e 30 de julho que continham os termos "hidroxicloroquina" ou "cloroquina" + "covid" ou "coronavírus". Além do uso de palavras-chave, o sistema de busca do CrowdTangle (que disponibiliza publicações e suas métricas de páginas e grupos monitorados pela ferramenta) também permite o uso de outros filtros na coleta de dados. Assim, filtramos apenas publicações que continham URLs, o que nos permitiu mapear a circulação do conteúdo na rede. No total, coletamos 25.747 publicações de páginas públicas e 45.204 publicações em grupos públicos.

A coleta de dados via CrowdTangle possui algumas limitações. O Facebook é proprietário e administrador da ferramenta, então acaba favorecendo métricas de desempenho social que reforçam as materialidades da própria plataforma - ou seja, métricas que favorecem as lógicas de datificação e os modelos de negócio do Facebook, dimensões centrais das plataformas digitais (D'ANDRÉA, 2020). Além disso, o CrowdTangle armazena apenas parte do conteúdo público do Facebook, sendo limitado a páginas e grupos monitorados pela plataforma. Ou seja, o que analisamos neste artigo não é o Facebook como um todo, mas uma amostra do conteúdo que circulou em páginas e grupos públicos monitorados pelo CrowdTangle. Apesar destas limitações, a ferramenta é útil para o nosso estudo porque disponibiliza dados em larga escala sobre a temática aqui analisada, o que nos permite mapear a circulação de URLs no Facebook.

Para a análise desses dados, utilizamos a Análise de Redes Sociais (WASSERMAN; FAUST, 1994). A partir das URLs e grupos ou páginas, criamos redes bipartidas com dois tipos de nós: (1) as URLs compartilhadas nas publicações; e (2) os grupos ou páginas que compartilharam as URLs. As arestas das redes indicam quando um grupo ou página compartilhou uma URL. Para identificar as "vizinhanças" em que as URLs foram compartilhadas, primeiro identificamos o maior componente conectado da rede, eliminando pequenas comunidades periféricas, ou seja, que estavam isoladas do centro da rede. Depois, utilizamos o cálculo de modularidade (BLONDEL et al., 2008), que é uma métrica de grupabilidade, ou seja, identifica os nós que estão mais próximos em função de suas conexões em comum (grupos ou páginas que compartilham as mesmas URLs formam pequenas comunidades). 
Para identificar os padrões de participação na circulação de conteúdo, utilizamos as métricas de grau ponderado de entrada e de saída. As métricas servem para identificar a frequência que uma URL foi compartilhada na rede, ou seja, quantas conexões a URL recebeu, que são representadas pelo grau ponderado de entrada; e a frequência que grupos ou páginas compartilharam URLs, isto é, quantas conexões os grupos e páginas enviaram, que são representadas pelo grau ponderado de saída.

Além de identificar as dinâmicas de participação de grupos e páginas na circulação de conteúdo relacionado a hidroxicloroquina, as métricas de grau também nos permitiram identificar as URLs que mais circularam em cada grupo (identificados por meio do cálculo de modularidade). Utilizamos a Análise de Conteúdo (KRIPPENDORFF, 2013) para analisar as URLs mais compartilhadas em cada rede. A distribuição do número de compartilhamentos das URLs seguiu um padrão de cauda longa: poucas URLs foram muito compartilhas e muitas URLs foram pouco compartilhadas (Figuras 1 e 2).

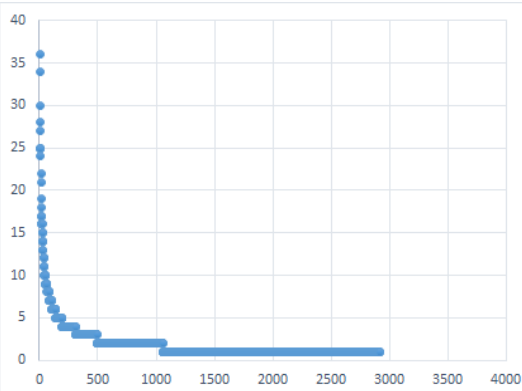

Figura 1. Distribuição de compartilhamento de URLs em páginas

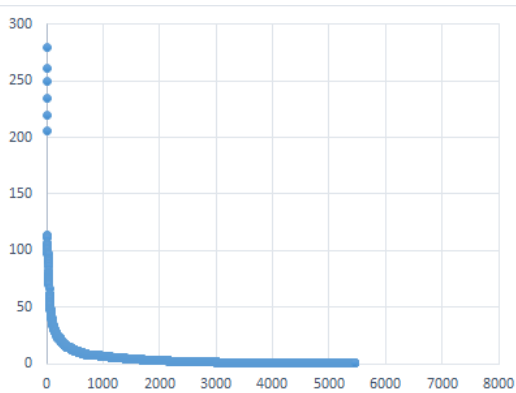

Figura 2. Distribuição de compartilhamento de URLs em grupos

Assim, decidimos realizar um recorte arbitrário e analisar as 50 URLs mais compartilhadas (maior grau ponderado de entrada) por cada um dos grupos identificados nas redes de páginas e grupos. No total, analisamos 400 URLs e identificamos: (1) a fonte da informação, ou seja, para que tipo de site o usuário seria direcionado ao clicar na URL; e (2) a presença de desinformação. Três analistas independentes classificaram todas as 400 URLs.

Para as fontes de informação, houve concordância média de mais de 95\% entre os analistas, alcançando um Krippendorff's Alpha de 0,914. Dividimos as fontes de informação em: imprensa, para veículos de jornalismo; mídias 
hiperpartidárias, conforme a definição do nosso referencial teórico; mídia social, para URLs que compartilhavam publicações no Facebook, vídeos no YouTube etc.; e institucional, para páginas institucionais (do governo, de organizações sociais, empresas etc.).

Para a desinformação, a concordância foi de $88 \%$ e o Alpha de 0,702. Para identificar o conteúdo desinformativo, realizamos buscas em fontes confiáveis, como imprensa, veículos de fact-checking e páginas institucionais (como Organização Mundial da Saúde). Em alguns casos, a desinformação era uma distorção de informações reais, o que explica a concordância inferior aos tipos de fonte, já que, além do acesso a informação confiável, dependia também da interpretação dos analistas. De qualquer forma, o Alpha alcançado garante o alto índice de concordância entre os três analistas. Além disso, para a classificação final, utilizamos a concordância entre pelo menos dois analistas (tanto para tipo de fonte, quanto presença de desinformação).

\section{Resultados}

Para a análise dos dados, criamos duas redes bipartidas, uma para circulação de URLs em páginas públicas e a outra em grupos públicos. Na visualização das redes, as URLs foram identificadas em azul e páginas e grupos, em vermelho. A representação das redes em formato de grafo pode ser vista nas figuras abaixo (3 e 4). Também destacamos as métricas gerais da rede (Tabela 1 ). 


\begin{tabular}{|c|c|c|}
\hline Métricas & Rede das páginas & Rede dos Grupos \\
\hline Número de nós & 20.884 & 16.986 \\
\hline Número de URLs & 16.845 & 12.115 \\
\hline Número de arestas & 24.899 & 44.696 \\
\hline Grau ponderado médio & 1,238 & 2,904 \\
\hline
\end{tabular}

Tabela 1. Métricas das redes

No total, o número de grupos e páginas é semelhante. Temos 4.039 páginas que compartilharam 16.845 URLs únicas; e 4.871 grupos que compartilharam 12.115 URLs únicas. Apesar da semelhança nos números, identificamos que os grupos foram mais ativos no compartilhamento de URLs, já que temos quase o dobro de arestas e grau ponderado médio mais alto. Então, ainda que seja menor o número de URLs únicas em comparação com as páginas (12.115 e 16.845), elas foram mais compartilhadas em grupos. Ou seja, o número de publicações com alguma das URLs sobre a hidroxicloroquina foi maior em grupos públicos do que em páginas. Isso também tem reflexo no maior componente conectado e na visualização da rede. Como pode ser visto nas figuras 3 e 4, há maior dispersão na rede de páginas, enquanto há maior centralidade na rede de grupos. O mesmo é evidenciado pelo número de componentes na rede, que são 1770 na rede de páginas e 461 na rede de grupos. Isto é, na rede de páginas há maior número de clusters isolados.

Em função dessas estruturas, optamos por olhar para o maior componente de cada uma das redes. Com base nesses componentes, realizamos cálculos de modularidade e identificamos as principais "vizinhanças" no compartilhamento de URLs sobre a hidroxicloroquina. As figuras 5 e 6 mostram a visualização dos grupos mais centrais nas redes analisadas. A tabela 2 descreve as métricas gerais das redes.

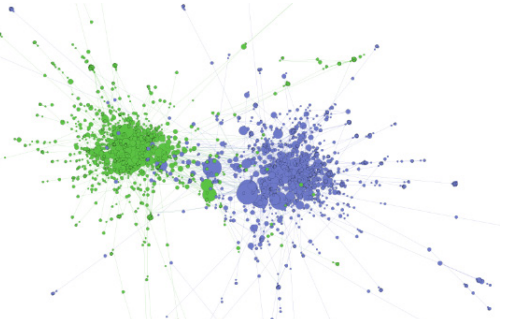

Figura 5. Rede das páginas

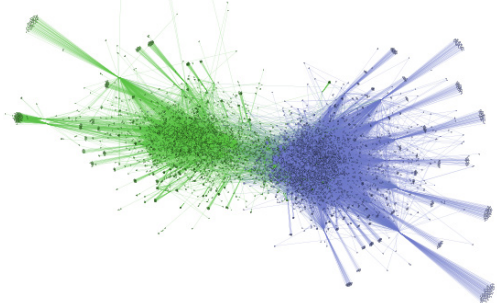

Figura 6. Rede dos grupos 


\begin{tabular}{|c|c|c|}
\hline Métricas & Rede das páginas & Rede dos Grupos \\
\hline Número de nós & 3.804 & 7.612 \\
\hline Número de URLs & 2.915 & 5.449 \\
\hline Número de arestas & 5.101 & 22.432 \\
\hline Grau ponderado médio & 1,368 & 3,217 \\
\hline
\end{tabular}

Tabela 2. Métricas dos clusters principais

As tendências na métrica de grau ponderado ficam ainda mais evidentes quando observados os grupos principais das redes. Há maior atividade no compartilhamento de URLs entre os grupos, que possuem grau ponderado médio quase três vezes maior do que as páginas. Nas duas redes encontramos alguns padrões semelhantes. Há polarização no compartilhamento de URLs, como podemos observar na estrutura dividida da rede (HIMELBOIM et al., 2017), ou seja, com dois clusters que possuem muitas conexões internas, mas poucas conexões externas. Isso quer dizer que as URLs que circularam em uma "vizinhança" não circularam na outra. Além disso, a partir da análise das URLs que mais circularam em cada grupo (discutida abaixo), identificamos que o cluster representado em verde (nas duas redes) possui um posicionamento contrário ao uso da hidroxicloroquina no tratamento do Covid-19, enquanto o cluster azul defende o uso do medicamento, que é apontado como cura ou profilaxia para a doença. Assim, as páginas e grupos que compartilham informações contrárias ao uso da hidroxicloroquina não compartilham conteúdo favorável ao seu uso. Da mesma forma, páginas e grupos que compartilham URLs favoráveis ao uso da hidroxicloroquina não compartilham informações contrárias.

O cenário é problemático, pois favorece uma exposição seletiva (STROUD, 2010) no consumo de informações dos usuários do Facebook sobre a hidroxicloroquina. Aqueles que recebem conteúdo de páginas e participam de grupos que compartilham conteúdo favorável ao uso do medicamento possuem menor tendência de ter contato com informações contrárias. Faz-se essencial analisar o comportamento no compartilhamento de URLs de cada cluster, já que tendem a compartilhar conteúdos distintos. Abaixo, destacamos as métricas de cada cluster em cada rede (Tabela 3). Em função do compartilhamento de informações em cada grupo, optamos por chamar o cluster verde de anti-hidroxicloroquina e o cluster azul de pró-hidroxicloroquina. 


\begin{tabular}{|c|c|c|c|c|}
\hline Métricas & \multicolumn{2}{|c|}{ Rede das páginas } & \multicolumn{2}{c|}{ Rede dos Grupos } \\
\hline Cluster & Anti-HCQ & Pró-HCQ & Anti-HCQ & Pró-HCQ \\
\hline Número de nós & 1.722 & 2.082 & 3.578 & 4.034 \\
\hline Número de URLs & 1.329 & 1.586 & 2.660 & 2.789 \\
\hline Número de arestas & 2.262 & 2.745 & 7.632 & 13.999 \\
\hline Grau ponderado médio & 1,335 & 1,349 & 2,26 & 3,863 \\
\hline
\end{tabular}

Nas duas redes, o cluster pró-hidroxicloroquina é mais ativo no compartiIhamento de URLs. A diferença é pequena na rede das páginas, mas é bem mais evidente na rede dos grupos. Isso quer dizer que o compartilhamento de URLs favoráveis ao uso do medicamento no combate ao Covid-19 foi especialmente prevalente em grupos públicos, quando comparados com páginas públicas, e também em comparação com grupos do cluster contrário ao uso do medicamento. Foram 18.403 publicações de URLs em 1.245 grupos públicos identificados no cluster pró-hidroxicloroquina. O número de compartilhamentos é quase o dobro do que ocorreu nos 918 grupos públicos identificados no cluster anti-hidroxicloroquina (10.120). No contexto das páginas, os números são mais próximos: 3.072 publicações em 496 páginas do cluster pró-HCQ e 2.664 publicações nas 393 páginas do cluster anti-HCQ.

Também analisamos as 50 URLs mais compartilhadas em cada cluster. Em função das dinâmicas de compartilhamento discutidas acima, as URLs mais compartiIhadas na rede dos grupos (entre 280 e 25 vezes) alcançaram maiores números de compartilhamento em comparação com as URLs da rede das páginas (entre 36 e 6). As figuras 7-10 detalham a distribuição no compartilhamento das 50 URLs mais centrais em cada cluster de cada rede. Enquanto as linhas de distribuição das páginas são semelhantes, vemos diferenças no caso dos grupos, em que o cluster pró-HCQ apresenta números superiores de compartilhamentos.
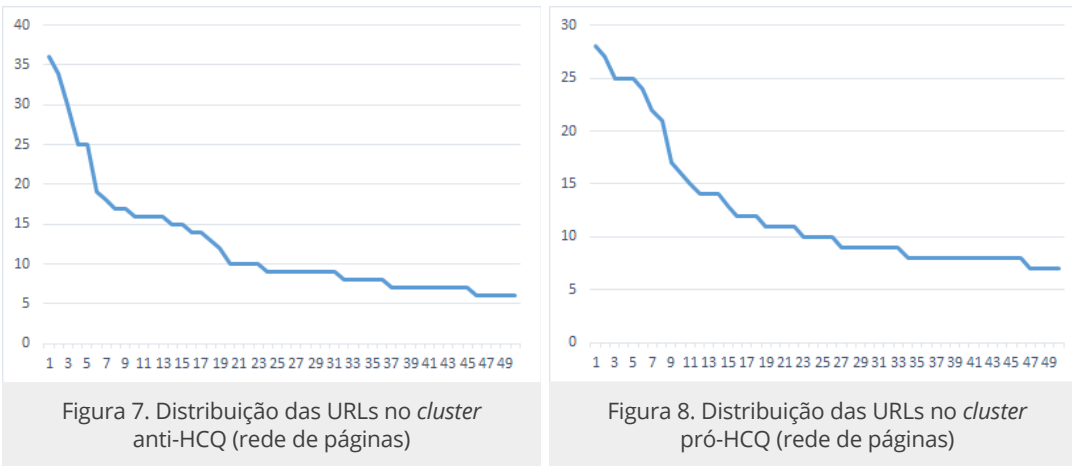


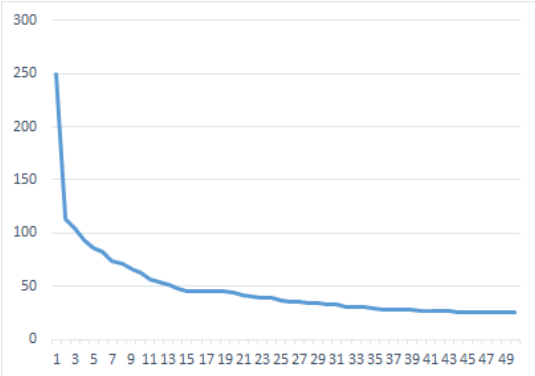

Figura 9. Distribuição das URLs no cluster anti-HCQ (rede de grupos)

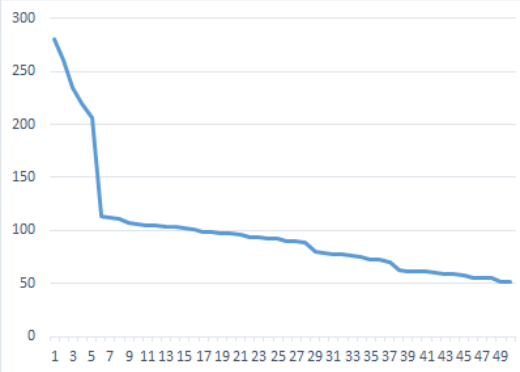

Figura 10. Distribuição das URLs no cluster pró-HCQ (rede de grupos)

Identificamos tendências bastante distintas entre os clusters na circulação de conteúdo. Enquanto o anti-hidroxicloroquina dá preferência a veículos de imprensa e não compartilha desinformação sobre o tema discutido, o cluster pró-hidroxicloroquina está mais associado ao compartilhamento de veículos hiperpartidários e de desinformação sobre o tema. Tais tendências aparecem tanto na rede de páginas quanto na rede de grupos. A tabela abaixo detalha a análise de conteúdo realizada a partir das 50 URLs mais compartilhadas.

\begin{tabular}{|c|c|c|c|c|c|c|c|c|}
\hline Rede & \multicolumn{4}{|c|}{ Rede das páginas } & \multicolumn{4}{c|}{ Rede dos Grupos } \\
\hline Cluster & \multicolumn{2}{|c|}{ Anti-HCQ } & \multicolumn{2}{|c|}{ Pró-HCQ } & \multicolumn{2}{|c|}{ Anti-HCQ } & \multicolumn{2}{c|}{ Pró-HCQ } \\
\hline & Freq. & Des. & Freq. & Des. & Freq. & Des. & Freq. & Des. \\
\hline Imprensa & 48 & 0 & 16 & 1 & 38 & 0 & 13 & 0 \\
\hline Hiperpartidário & 1 & 0 & 28 & 22 & 11 & 0 & 32 & 25 \\
\hline Mídia social & 1 & 0 & 1 & 1 & 0 & 0 & 2 & 2 \\
\hline Institucional & 0 & 0 & 5 & 1 & 1 & 0 & 3 & 1 \\
\hline Total & $\mathbf{5 0}$ & $\mathbf{0}$ & $\mathbf{5 0}$ & $\mathbf{2 5}$ & $\mathbf{5 0}$ & $\mathbf{0}$ & $\mathbf{5 0}$ & $\mathbf{2 8}$ \\
\hline
\end{tabular}

Tabela 4. URLs mais compartilhadas

A primeira coisa que notamos é a diferença no conteúdo que circula em páginas e grupos. Comparamos as URLs mais compartilhadas em cada rede. No cluster anti-hidroxicloroquina, 17 URLs apareceram entre as mais compartilhadas em páginas e grupos. As outras 66 estavam entre as mais compartilhadas apenas em um dos contextos. No pró-hidroxicloroquina, o número de URLs em comum foi 23, enquanto 54 apareceram em apenas uma das redes. Assim, vemos que o conteúdo compartilhado em páginas e grupos possuem distinções, o que reflete também nos resultados relacionados com a análise das fontes e da desinformação. 
Ainda que nenhuma URL do cluster anti-HCQ tenha sido marcada como desinformativa, observamos diferenças entre as fontes em cada uma das redes. Na rede das páginas, 48 URLs são oriundas de veículos da imprensa, enquanto na rede de grupos, esse número se reduz para 38. O inverso ocorre com as mídias hiperpartidárias, que aparecem em apenas uma URL nas páginas anti-HCQ e em 11 URLs nos grupos. Ainda que as URLs não reproduzam desinformação, percebemos um contexto mais partidário nos grupos em comparação com as páginas. A mesma tendência aparece no cluster próhidroxicloroquina, representando também um aumento da desinformação. A principal distinção, porém, se dá na comparação entre clusters favoráveis e contrários ao uso do medicamento. Nenhuma das URLs compartilhadas pelas páginas e nos grupos do cluster anti-HCQ apresenta desinformação. Já no cluster pró-HCQ, metade das URLs compartilhadas por páginas são desinformativas e o número é ainda maior no contexto dos grupos (56\%). Além disso, também vemos maior presença de mídias hiperpartidárias no cluster pró-HCQ, tanto na rede de páginas quanto de grupos. Assim, observamos uma tendência assimétrica na polarização entre os clusters (BENKLER, FARIS; ROBERTS, 2018), já que um compartilha principalmente conteúdo da imprensa e o outro dá preferência a veículos hiperpartidários e conteúdo desinformativo.

Dentre as URLs desinformativas, identificamos compartilhamentos de chamadas como: "E viva a Cloroquina! A cura em casa sem sequer utilizar a rede hospitalar" (Jornal da Cidade Online) ${ }^{11}$, que afirma que o medicamento deve ser utilizado nos primeiros dias da doença e seria o tratamento mais eficaz; "A militância médica esquerdista por trás da pesquisa com alta dosagem de cloroquina em Manaus" (Conexão Política) ${ }^{12}$, que afirma que petistas teriam organizado estudo clínico para desqualificar a droga; e "Com uso da cloroquina, Bolsonaro testa negativo para Covid-19", que afirma que Bolsonaro seria "a prova viva da eficácia do remédio" (Jornal da Cidade Online) ${ }^{13}$, já que o político realizou tratamento com HCQ e não teve sintomas graves. As três URLs estão entre as mais compartilhadas em páginas e grupos do cluster pró-HCQ.

11 <https://www.jornaldacidadeonline.com.br/noticias/19727/e-viva-a-cloroquina-a-cura-em-casa-sem-sequer-utilizar-a-rede-hospitalar>. Acessado em 23 de fevereiro de 2021.

12 <http://conexaopolitica.com.br/ultimas/a-militancia-medica-esquerdista-por-tras-da-pesquisa-com-alta-dosagem-de-cloroquina-em-manaus/>. Acessado em 23 de fevereiro de 2021.

13 <https://www.jornaldacidadeonline.com.br/noticias/21974/com-uso-da-cloroquina-bolsonaro-testa-negativo-para-covid-19>. Acessado em 23 de fevereiro de 2021. 
Mesmo quando apareciam veículos de imprensa nas páginas e grupos do cluster pró-HQC, o conteúdo das notícias geralmente trazia um enquadramento positivo para a hidroxicloroquina. Por exemplo, diversas notícias relatavam a liberação do uso do medicamento (pelo Ministério da Saúde, prefeituras etc.) e declarações de pessoas que se recuperaram da doença. Nesses casos, o jornalismo "declaratório" favoreceu uma narrativa pró-HQC e pode ter auxiliado na formação de percepções equivocadas sobre a eficácia da droga.

Também identificamos poucas fontes que produziam conteúdo científico ou especializado no campo da medicina ou de fármacos. Apenas uma URL do cluster anti-HCQ na rede de grupos é oriunda da plataforma científica The Lancet. Já no cluster pró-HQC, a Associação Médica Brasileira aparece tanto nas páginas quanto grupos e o grupo farmacêutico Novartis aparece entre as URLs que mais circularam nos grupos. Vemos, portanto, que o enquadramento da discussão não foi pautado diretamente por fontes especializadas. Ainda assim, apareciam informações relacionadas a estudos científicos e recomendações de especialistas, porém por meio da mediação da imprensa.

Em função das dinâmicas de circulação de cada rede e de cada cluster, também identificamos a relevância das URLs desinformativas que foram compartilhadas pelo cluster pró-hidroxicloroquina, principalmente em grupos. A tabela abaixo detalha a frequência no compartilhamento das URLs analisadas em cada cluster.

\begin{tabular}{|c|c|c|c|c|}
\hline Rede & \multicolumn{2}{|c|}{ Rede das páginas } & \multicolumn{2}{c|}{ Rede dos Grupos } \\
\hline Cluster & Anti-HCQ & Pró-HCQ & Anti-HCQ & Pró-HCQ \\
\hline Desinformação & 0 & 316 & 0 & 2509 \\
\hline Outro & 613 & 299 & 2092 & 2416 \\
\hline Total & $\mathbf{6 1 3}$ & $\mathbf{6 1 5}$ & $\mathbf{2 0 9 2}$ & $\mathbf{4 9 2 5}$ \\
\hline
\end{tabular}

Tabela 5. Frequência do compartilhamento de URLS

A partir dos dados, podemos perceber o impacto de questões já destacadas na análise das métricas de redes. O alto grau ponderado médio do cluster pró-HCQ na rede de grupos tem reflexos na frequência de compartilhamento de desinformação. Na comparação entre os dois clusters na rede de grupos, apenas o conteúdo desinformativo do pró-HCQ (28 URLs) foi compartilhado com maior frequência do que todas as 50 URLs mais compartilhadas naquele anti-HCQ. No contexto das páginas, a frequência total é semelhante entre os dois clusters. 
A análise também evidencia o papel dos grupos no compartilhamento de desinformação. O total de desinformação compartilhado no cluster próHCQ na rede de grupos é quase oito vezes maior em comparação com a desinformação compartilhada pelo mesmo cluster na rede de páginas. Ainda que páginas possam ter um alcance maior do que grupos, vemos que as interações que ocorrem dentro de grupos públicos podem motivar o espalhamento de desinformação no Facebook.

Nesse contexto, decisões do Facebook, como reduzir alcance de veículos de jornalismo e dar preferência a publicações dos amigos de cada usuário na plataforma ${ }^{14}$, podem ter efeitos nas dinâmicas que identificamos. $\mathrm{O}$ compartilhamento de desinformação em grupos públicos, em publicações que depois podem ser compartilhadas por membros dos grupos e seus contatos, pode ser motivado por um processo aparentemente mais orgânico na circulação de informações. Isto é, pode ocorrer como estratégia que visa uma apropriação das lógicas de circulação de conteúdo no Facebook para dar maior visibilidade para links desinformativos. Ou seja, as lógicas da plataforma e seus processos de filtragem de conteúdo (GILLESPIE, 2018; D'ANDRÉA, 2020) podem estar favorecendo um movimento de compartilhamento de desinformação em grupos. Além disso, ao reduzir a visibilidade de veículos de imprensa, o conteúdo que desmente a desinformação pode acabar limitado a páginas dos próprios veículos.

Esta diferença entre páginas e grupos também parece estar relacionada com as affordances da plataforma (EVANS et al., 2017; BUCHER; HELMOND, 2018). Enquanto páginas são gerenciadas por apenas um ou um número limitado de usuários, os grupos possibilitam que todos os membros postem qualquer tipo de conteúdo. Assim, a maior frequência no compartilhamento de URLs, particularmente de URLs desinformativas, pode ter a ver com a maior abertura para a publicação de usuários em geral.

Destaca-se, ainda, que o contexto observado pode ter sido influenciado pela forma como os usuários percebem esses espaços (VALERIANI; VACCARI, 2018). Mesmo que os grupos analisados sejam públicos, pode haver maior percepção de privacidade entre os usuários, na comparação com as páginas. Além disso, muitos desses grupos podem ser focados em temáticas específicas, o que favorece um contexto coletivo de homofilia. Assim, os usuários 
podem perceber o grupo como um lugar mais propício para o compartilhamento de conteúdo de mídias hiperpartidárias (como visto em ambos os clusters) e de desinformação (como visto no cluster pró-HCQ).

De forma geral, identificamos redes divididas (HIMELBOIM et al., 2017) tanto na circulação de URLs em páginas, quanto grupos. Essa estrutura favorece a polarização de sentimentos relacionados à pandemia (ALLCOTT et al., 2020) e pode favorecer a exposição seletiva apenas a conteúdos que favorecem uma narrativa em particular (STROUD, 2010). Como vimos, isso ocorre mesmo quando a imprensa aparece com maior centralidade no cluster pró-hidroxicloroquina, geralmente em contextos em que são reproduzidas declarações favoráveis ao uso do medicamento ou informações sobre sua liberação para o uso.

Também identificamos assimetrias entre os clusters em ambas as redes (BENKLER, FARIS; ROBERTS, 2018). Enquanto o anti-HCQ não compartilhou desinformação e deu preferência a veículos de imprensa, o cluster pró-HCQ esteve associado ao espalhamento de desinformação e deu maior centralidade a mídias hiperpartidárias. Assim, percebemos uma ação das páginas e dos grupos para dar preferência a um tipo de conteúdo específico como forma de reforço de uma posição sobre a discussão, mesmo que o conteúdo contenha informações falsas.

Notamos ainda diferenças no tipo de conteúdo, nas fontes de informação e no comportamento de compartilhamento de URLs em páginas e grupos. Percebemos maior centralidade de veículos hiperpartidários e de desinformação na rede de grupos. Além disso, identificamos que o cluster pró-HCQ na rede de grupos foi especialmente ativo no compartilhamento de desinformação, atingindo frequência bastante superior ao outro grupo e aos clusters da rede de páginas. Essas diferenças podem ser reflexo tanto das affordances de cada espaço, quanto da forma como os usuários percebem estes espaços (EVANS et al., 2017; BUCHER; HELMOND, 2018; VALERIANI; VACCARI, 2018).

Os nossos resultados possuem algumas implicações. A divisão nas redes favorece a exposição seletiva (STROUD, 2010). Com isso, torna-se mais difícil a circulação de conteúdo verificado e de veículos de fact-checking nos espaços que circulam desinformação. Ou seja, há maior tendência de que o conteúdo verificado circule majoritariamente entre páginas e grupos que não compartilham a desinformação que foi checada, enquanto as páginas e grupos que estão compartilhando desinformação possuem a tendência de rejeitar a circulação do conteúdo verificado. 
As altas frequências no compartilhamento de desinformação nos grupos também possuem impacto nas formas de combate ao conteúdo problemático no Facebook. Ainda que a plataforma tenha iniciativas de marcar publicações de páginas como conteúdo desinformativo ou mesmo remover o conteúdo, como fez com declaração falsa de Jair Bolsonaro sobre a hidroxicloroquina ${ }^{15}$, os nossos resultados apontam para a necessidade de monitorar grupos públicos. Como vimos, a maior frequência de conteúdo desinformativo foi compartilhado nestes espaços. Além disso, entre os nossos dados, haviam três publicações desinformativas de Bolsonaro na própria plataforma (duas na rede grupos e uma na rede de páginas). Mesmo com conteúdo distorcido, as publicações seguem disponíveis na plataforma.

Recentemente, o Facebook removeu páginas do movimento QAnon, que espalhava teorias da conspiração principalmente nos Estados Unidos ${ }^{16}, 0$ que mostra a capacidade do Facebook de identificar e filtrar o conteúdo problemático que circula na plataforma. Assim, são necessárias ações mais incisivas no combate à desinformação, como reduzir a circulação de veículos hiperpartidários na plataforma, marcar ou excluir URLs com informações distorcidas ou falsas e monitorar o conteúdo desinformativo publicado em grupos públicos, que podem estar servindo para mobilizar a circulação de desinformação na plataforma.

Por fim, observamos que frequentemente as URLs de veículos de imprensa no cluster pró-HCQ seguiam uma prática de jornalismo "declaratório". Ao apenas relatar declarações de supostas curas por meio da hidroxicloroquina, o jornalismo acaba contribuindo para o fortalecimento de narrativas desinformativas. Assim, cabe ao jornalismo ser mais crítico no processo de produção de notícias, visando uma posição de combate à desinformação.

\section{Considerações finais}

Neste artigo, buscamos explorar a circulação de desinformação sobre a hidroxicloroquina no Facebook, particularmente visando compreender a circulação de desinformação sobre o tema. Para isso, coletamos mais de 70 mil publicações de páginas e grupos públicos via CrowdTangle (2020). Utilizamos a Análise de Redes Sociais para mapear as redes de circulação

15 <https://www1.folha.uol.com.br/poder/2020/04/facebook-apagou-post-de-bolsonaro-por-alegacao-falsa-de-cura-para-coronavirus.shtml>. Acessado em 15 de novembro de 2020.

16 <https://www.uol.com.br/tilt/noticias/reuters/2020/10/06/facebook-remove-grupos-qanon-ao-classificar-movimento-como-perigoso.htm>. Acessado em 23 de fevereiro de 2021. 
de URLs nas páginas e nos grupos. Utilizamos a Análise de Conteúdo para analisar as fontes de informação e a presença de desinformação nas URLs que mais circularam em cada cluster identificado nas redes.

A partir disso, identificamos que (1) a circulação de conteúdo sobre a hidroxicloroquina no Facebook se dá de forma polarizada, tanto no contexto de páginas, quanto de grupos. Este contexto favorece a exposição seletiva e a desinformação e indica que as URLs que circulam em um dos clusters não circulam no outro. (2) O conteúdo desinformativo identificado reforça uma narrativa pró-hidroxicloroquina e, portanto, circulou no cluster pró-HCQ. Assim, a circulação de desinformação foi assimétrica, presente em mais de 50\% das URLs mais compartilhadas no cluster pró-HCQ. (3) Percebemos que grupos deram maior centralidade a veículos hiperpartidários em comparação com as páginas. Também identificamos mais URLs desinformativas nos grupos do cluster pró-HCQ do que nas páginas. Por fim, vimos que a frequência com que URLs são publicadas nos grupos foi bastante superior a páginas, tendo um impacto no número de compartilhamentos de URLs e, particularmente, de URLs informativas nos grupos do cluster pró-HCQ.

O nosso primeiro resultado (polarização) mostra a necessidade de encontrar formas de circular informações entre grupos opostos nas mídias sociais. Particularmente, também em relação ao nosso segundo resultado (assimetria), é necessário buscar estratégias para combater a desinformação, já que os grupos que a compartilham evitam o compartilhamento de conteúdo verificado. Por fim, o nosso terceiro resultado (diferenças entre páginas e grupos) aponta para a necessidade de considerar as particularidades das ferramentas no combate à desinformação. Além de estratégias como marcar ou remover conteúdo desinformativo de páginas públicas, é preciso desenvolver estratégias para frear o compartilhamento de desinformação em grupos no Facebook.

Esta pesquisa possui algumas limitações. Tivemos acesso apenas a dados oriundos do CrowdTangle, o que não compreende o Facebook como um todo. Além disso, foram necessários recortes nos dados para a identificação dos clusters principais, especialmente na rede das páginas, onde havia maior dispersão. Além disso, medimos apenas a frequência de compartilhamento de URLs nas publicações coletadas, porém não medimos o número de compartilhamento dessas publicações. Isso explica, por exemplo, os números mais baixos na frequência de URLs nas páginas. 
Felipe Bonow Soares é doutor em Comunicação e Informação pela Universidade Federal do Rio Grande do Sul; coordena o Laboratório de Pesquisa em Mídia, Discurso e Análise de Redes (MIDIARS).

fbonowsoares@gmail.com

Paula Viegas é doutoranda em Comunicação e Informação no PPGCOM/UFRGS e pesquisadora do Laboratório de Pesquisa em Mídia, Discurso e Análise de Redes (MIDIARS).

paularviegas@gmail.com

Carolina Bonoto é doutoranda em Comunicação e Informação no PPGCOM/UFRGS e pesquisadora do Laboratório de Pesquisa em Mídia, Discurso e Análise de Redes (MIDIARS).

c.bonoto@gmail.com

Raquel Recuero é professora e pesquisadora da Universidade Federal de Pelotas e do PPGCOM da Universidade Federal do Rio Grande do Sul; é doutora em Comunicação e Informação pela Universidade Federal do Rio Grande do Sul; coordena o Laboratório de Pesquisa em Mídia, Discurso e Análise de Redes (MIDIARS).

raquel.recuero@ufpel.edu.br

Contribuicões de cada autor: Felipe Bonow Soares supervisão e gestão do projeto de pesquisa, fundamentação teórica e conceituação, curadoria de dados, escrita (primeira redação, revisão e edição), metodologia, análise formal do corpus, gestão de software, construção de figuras e tabelas. Paula Viegas - fundamentação teórica e conceituação, escrita (revisão e edição), metodologia, análise formal do corpus. Carolina Bonoto - fundamentação teórica e conceituação, escrita (revisão e edição), metodologia, análise formal do corpus. Raquel Recuero 


\begin{abstract}
- supervisão e gestão do projeto de pesquisa, fundamentação teórica e conceituação, curadoria de dados, escrita (revisão e edição), metodologia, análise formal do corpus, obtenção de financiamento, gestão de software.
\end{abstract}

\title{
Referências
}

ALLCOTT, H.; BOXELL, L.; CONWAY, J. C.; GENTZKOW, M.; THALER, M.; YANG, D. Y. Polarization and Public Health: Partisan Differences in Social Distancing during the Coronavirus Pandemic, Working Paper 26946. Cambridge (EUA): National Bureau of Economic Research, 2020. Disponível em: <https://www.nber.org/system/files/working_papers/w26946/ w26946.pdf>. Acessado em 14 mar.2021.

ARAÚJO, R.; OLIVEIRA, T. A. Desinformação e mensagens sobre a hidroxicloroquina no Twitter. In: SciELO Pré-prints, 2020. Disponível em <https://revistas.ufpr.br/atoz/article/ view/75929>. Acessado em 14 mar.2021.

BAKSHY, E.; MESSING, S.; ADAMIC, L. A. Exposure to ideologically diverse news and opinion on Facebook. In: Science, v. 348, n. 6239, 2015, p. 1130-1132. Disponível em <DOI: 10.1126/ science.aaa1160>. Acessado em 14 mar.2021.

BARBERÁ, P. Social Media, Echo Chambers, and Political Polarization. In: PERSILY, N.; TUCKER, J. (org.). Social Media and Democracy: The State of the Field and Prospects for Reform, p. 34-55. Cambridge (ING): Cambridge University Press, 2020.

BENKLER, Y.; FARIS, R.; ROBERTS, H. Network Propaganda: Manipulation, disinformation, and radicalization in American politics. Nova York: Oxford University Press, 2018.

BLONDEL, V. D.; GUILLAUME, J.; LAMBIOTTE, R.; LEFEBVRE, E. Fast unfolding of communities in large networks. In: Journal of Statistical Mechanics: Theory and Experiment, Vol. 2008. Disponível em < https://iopscience.iop.org/article/10.1088/1742-5468/2008/10/ P10008/meta>. Acessado em 14 mar.2021.

BOYD, D. Social Network Sites as Networked Publics: Affordances, Dynamics, and Implications. In: PAPACHARISSI, Z. A Networked Self: Identity, Community, and Culture on Social Network Sites, p. 39-58. Nova York: Routledge, 2011.

BRENNEN, J. S.; SIMON, F. M.; NIELSEN, R. K. Beyond (Mis)Representation: Visuals in COVID-19 Misinformation. In: The International Journal of Press/Politics, Online First, 2020. Disponível em <https://doi.org/10.1177/1940161220964780>. Acessado em 14 mar.2021.

BUCHER, T.; HELMOND, A. The Affordances of Social Media Platforms. In: BURGESS, J.; MARWICK, A.; POELL, T. Poell (org.). The SAGE Handbook of Social Media, p. 233-253. Londres: Sage, 2018.

CROWDTANGLE TEAM. CrowdTangle, aplicativo de propriedade do Facebook. Menlo Park (EUA): Facebook, 2020. Disponível em <https://apps.crowdtangle.com/search/>. Acessado em 15 out. 2020. 
D'ANDRÉA, C. Pesquisando plataformas online: conceitos e método. Salvador: EDUFBA, 2020.

EVANS, S. K.; PEARCE, K. E.; VITAK, J.; TREEM, J. W. Explicating Affordances: a Conceptual Framework for Understanding Affordances in Communication Research. In: Journal of Computer-Mediated Communication, v. 22, n. 1, p. 35-52, 2017. Disponível em <https:// doi.org/10.1111/jcc4.12180>. Acessado em 14 mar.2021.

FALLIS, D. What Is Disinformation?. In: Library Trends, v. 63, n. 3, p. 401-426, 2015. Chicago (EUA): University of Illinois, 2015. Disponível em <https://doi.org/10.1353/lib.2015.0014>. Acessado em 14 mar.2021.

GILLESPIE, T. Custodians of the internet: platforms, content moderation, and the hidden decisions that shape social media. Yale (EUA): University Press, 2018.

GRUZD, A.; MAI, P. Going viral: How a single tweet spawned a COVID-19 conspiracy theory on Twitter. In: Big Data \& Society, v. 7, n. 2, p. 1-9, 2020. Disponível em <https://journals. sagepub.com/doi/pdf/10.1177/2053951720938405>. Acessado em 14 mar.2021.

HIMELBOIM, I.; SMITH, M. A.; RAINIE, L.; SCHNEIDERMAN, B.; ESPINA, C. Classifying Twitter Topic-Networks Using Social Network Analysis. In: Social Media + Society, p. 1-13, 2017. Disponível em <https://doi.org/10.1177/2056305117691545>. Acessado em 14 mar.2021.

KEARNEY, M. D.; CHIANG, S. C.; MASSEY, P. M. The Twitter origins and evolution of the COVID-19 "plandemic" conspiracy theory. In: The Harvard Kennedy School (HKS) Misinformation Review, 2020. Disponível em <https://doi.org/10.37016/mr-2020-42>. Acessado em 14 mar.2021.

KRIPPENDORF, K. Content Analysis: An Introduction to Its Methodology. Thousand Oaks (EUA): Sage Publications, 2013.

LARSSON, A. O. News Use as Amplification - Norwegian National, Regional and Hyperpartisan Media on Facebook. In: Journalism \& Mass Communication Quarterly, v. 96, p. 3, 2019. Disponível em <https://doi.org/10.1177/1077699019831439>. Acessado em 14 mar.2021.

NEWMAN, N.; FLETCHER, R.; SCHULZ, A.; ANDI, S.; NIELSEN, R. K. Reuters Institute Digital News Report 2020. Oxford (ING): Reuters Institute/Oxford University, 2020.

RECUERO, R.; BASTOS, M.; ZAGO, G. Análise de Redes para Mídia Social. Porto Alegre: Sulina, 2015.

RECUERO, R.; SOARES, F. O Discurso Desinformativo sobre a Cura do COVID-19 no Twitter: Estudo de caso. E-Compós, Ahead of Print, 2020. Disponível em <https://doi.org/10.30962/ ec.2127>. Acessado em 14 mar.2021.

RECUERO, R.; SOARES, F. B.; GRUZD, A. Hyperpartisanship, Disinformation and Political Conversations on Twitter: The Brazilian Presidential Election of 2018. Proceedings of the International AAAI Conference on Web and Social Media, vol. 14 (1), p. 569-578, 
2020. Disponível em <https://ojs.aaai.org/index.php//CWSM/article/view/7324/7178>. Acessado em 14 mar.2021.

RECUERO, R.; SOARES, F. B.; ZAGO, G. Polarization, Hyperpartisanship and Echo Chambers: How the disinformation about Covid-19 circulates on Twitter. SciELO Preprints, 2020. Disponível em <https://doi.org/10.1590/SciELOPreprints.1154>. Acessado em 14 mar.2021.

STROUD, N. J. Polarization and Partisan Selective Exposure. In: Journal of Communication, v. 60, n. 3, p. 556-576, 2010. Disponível em <https://doi.org/10.1111 /j.1460-2466.2010.01497.x>. Acessado em 14 mar.2021.

VALERIANI, A.; VACCARI, C. Political talk on mobile instant messaging services: a comparative analysis of Germany, Italy, and the UK. In: Information, Communication \& Society, v. 21, n. 11, p. 1715-1731, 2018. Disponível em <https://doi.org/10.1080/136911 8X.2017.1350730>. Acessado em 14 mar.2021.

WARDLE, C.; DERAKHSHAN, H. Information disorder: Toward an interdisciplinary framework for research and policy making. Strasbourg (FRA): Council of Europe, 2017.

WASSERMAN, S.; FAUST, K. Social Network Analysis: Methods and Appliccations. Cambridge (ING): Cambridge University Press, 1994.

XAUDIERA, S.; CARDENAL, A. S. Ibuprofen narratives in five European countries during the COVID-19 pandemic. In: The Harvard Kennedy School (HKS) Misinformation Review, 2020. Disponível em <https://doi.org/10.37016/mr-2020-029>. Acessado em 14 mar.2021.

ZAGO, G.; BASTOS, M. T. Visibilidade de Notícias no Twitter e no Facebook: Análise Comparativa das Notícias mais Repercutidas na Europa e nas Américas. Brazilian Journalism Research, v. 9, n. 1, p. 116-133, 2013. Disponível em <https://bjr.sbpjor.org.br/bjr/article/ view/510>. Acessado em 14 mar.2021.

Artigo recebido em 16/11/2020 e aprovado em 25/02/2021. 